Gut, 1983, 24, 940-945

Liver and biliary

\title{
Primary biliary cirrhosis: geographical clustering and symptomatic onset seasonality
}

\author{
A N HAMLYN, A F MACKLON, AND O JAMES \\ From the University Departments of Medicine and Geriatrics, Freeman Hospital, Newcastle-upon-Tyne
}

SUMmaRY Patients with primary biliary cirrhosis (primary non-suppurative destructive cholangitis) in the north east region of England were studied over a five year period and, to evaluate epidemicity, compared with two contemporaneous disease series of known occurrence. These were: terminal renal failure, all causes (low or absent epidemicity $n=106$ ) and an outbreak of echovirus 19 disease (high epidemicity $n=201$ ). Eight primary biliary cirrhosis-affected men and 109 women from an estimated catchment population of 2.08 million were identified. The current diagnosis rate was $1.0 / 100000(1.8 / 100000$ for women of 15 or more). There were 18 deaths, mean survival from diagnosis 4.0 years. Within the region prevalence varied from $3 \cdot 7 / 100000$ in rural areas to $14.4 / 100000$ in industrial urban areas. In the conurbation, prevalence rates varied insignificantly. Here, most cases were concentrated in central districts, where the proportion of asymptomatic presentations was $50 \%$. Outside the conurbation the asymptomatic proportion fell to $21 \%$, suggesting low incidental diagnosis rates. When compared with echovirus 19 , primary biliary cirrhosis was of low or absent epidemicity, and similar to renal failure in its uniform geographical distribution and lack of clustering. Forty three patients (37\% of the total), however, had significantly seasonal symptomatic presentations $(p<0.01)$, although scan statistic testing failed to show clustering of onset in time. Apparently provocative factors associated with primary biliary cirrhosis symptomatic onset were identified in only $11(9.4 \%)$ of patients. Age-specific onset rates rose linearly between ages 35 and 65, and nearly one third of patients presented after 65 years, two thirds of deaths occurring in this age group. There is thus no evidence in north east England of geographical anomalies in the distribution of primary biliary cirrhosis. International differences may be partly explained by environmental factors influencing seasonal presentation, such as sunlight. Diagnosis rates are profoundly influenced by increased medical awareness, especially in the elderly, of this now relatively common disease and increased use of the mitrochondrial (AMA) antibody test.

Primary biliary cirrhosis (primary non-suppurative destructive cholangitis) chiefly affects middle aged or elderly women and is characterised by progressive destruction of intrahepatic bile ducts. After a variable period of increasing cholestasis biliary cirrhosis develops, resulting finally in death from hepatic failure or the consequences of portal hypertension. ${ }^{12}$ The causation of primary biliary cirrhosis and the reason for its female predilection

Address for correspondence: Dr A N Hamlyn. Gastrointestinal Unit. Wordsley Hospital. Stourbridge. West Midlands DY8 5QX.

Received for publication 14 January 1983 are unknown and little is known of its distribution and occurrence in the general population. This paper describes some epidemiological attributes of a series of patients diagnosed in Newcastle-upon-Tyne and its surrounding region.

\section{Methods}

PATIENTS AND CONTROLS

The study was centred upon Newcastle-upon-Tyne, a large industrial conurbation with an extensive hinterland. Recruitment of primary biliary cirrhosis diagnoses was maximised by collection over a five 
year period from three sources: (1) a register of patients identified through personal enquiry at district general hospitals in Durham, Tyne and Wear, Northumberland, and North and South Tyneside. (2) Investigation of incidentally discovered mitochondrial antibody positivity. (3) Office of Population Censuses and Survey death certification data. Patients were interviewed and examined by at least one of the authors; death certificates were validated by comparison with the original case record.

The population base was defined by reference to postcode data; age and sex structure was obtained from census data. It was estimated to comprise 2.08 million, of whom 1.08 were females of age 15 or over.

\section{CRITERIA}

Those of the International Association of the Study of the Liver ${ }^{3}$ were used for the diagnosis of primary biliary cirrhosis: biochemical evidence of chronic cholestasis and diagnostic or compatible liver biopsy histology, together with mitochondrial antibody titre $1 / 20$ or greater. Where appropriate bile duct patency was established radiographically.

\section{MITOCHONDRIAL ANTIBODY}

Detection was by incubating patient serum at an initial dilution of $1 / 10$ with mouse kidney cryostat sections. Behring goat fluorescent polyspecific conjugate was used at a working dilution of $1 / 30$ and a Zeiss Orthoplan microscope used to detect UV immunofluorescence. ${ }^{4}$

\section{CONTROLS}

Comparison was made with two sets of controls. These were selected to reflect local referral patterns and comprised a well documented outbreak of Echovirus 19 disease from the same area (highly infective, high epidemicity controls $n=201)^{5}$ as well as a series of patients referred from the region over the study period for dialysis treatment of terminal renal failure, all causes (demonstrably non-infective, low epidemicity controls $n=106$ ) at the Newcastle University Hospitals.

To avoid problems due to non-uniform population distribution, geographical and epidemiological data were analysed using home address postcodes. ${ }^{6}$ The Post Office provided computer listings of 1978 household (small user) delivery points enumerated by postal area, district, and sector. Thus, with the aid of census data ${ }^{78}$ giving household composition, estimates could be made of the catchment area population. Statistical methods included Mantel's $\chi^{29}$ and clustering was analysed using $\chi^{2}$-based ${ }^{1011}$ and scan ${ }^{12}$ statistics. Seasonality was tested with the non-parametric Kuiper statistic. ${ }^{13}$

\section{Results}

\section{SEX RATIO}

There were eight men and 109 women in the sample, yielding a male-female sex ratio of $1: 14$. This compares with $1: 1 \cdot 1$ in the base population over 15 years of age $(p<0.001)$.

\section{ORIGIN}

A pattern of low migration into the population base was shown, $15(12.8 \%)$ stated birthplace outside the North East. All were ethnic Europeans and had spent most, or all of their lives in the Newcastle area.

\section{FREQUENCY}

Of 117 patients, $103(88 \%)$ presented after 1972, a mean diagnosis rate of $12 \cdot 3$ /year. All but two (98\%) gave addresses at diagnosis within Newcastle or its neighbouring counties. By 1977 diagnosis rate had risen to $19 /$ year, nearly $1 / 100000$ for the catchment population or $1.8 / 100000$ for women aged 15 or over. There were 18 deaths in the series, median survival 4.0 years.

\section{SUB-REGIONAL VARIATIONS}

Considerable variation in point-prevalence rate occurred within the region. In general, high prevalence rates were found in densely populated areas (25 cases/hectare) and lowest rates $(<1 /$ hectare) in rural areas at greatest distance from the main Newcastle hospitals. Variation within the conurbation was insignificant $\left(\chi^{2}=5 \cdot 90, p<0 \cdot 10\right)$. Nonetheless, strikingly low rates $(<8 /$ hectare) were seen in some urban districts outside Newcastle. In these the proportion of asymptomatic presentations to total $(21 \%)$ was significantly lower than in the city of Newcastle $(50 \%, \mathrm{p}<0.05)$.

\section{EPIDEMICITY AND SEASONALITY}

Of all the prospectively interviewed patients, 43 (37\%) were able to date symptomatic onset (jaundice, pruritis, abdominal pain, malaise) to within a particular month of the year. Stated symptomatic onset tended to cluster in the spring and early summer with Kuiper's test confirming significant seasonality $(\mathrm{p}<0 \cdot 01)$. Seasonality testing was strongly positive for echovirus 19 onset $(p<0 \cdot 001)$ and negative for renal failure onset as judged by first dialysis $(p<0.7)$. The scan statistic (analysing clustering over the years, Fig. 1), however, yielded an insignificant value for primary biliary cirrhosis onset $(p<0.09)$, as in the case of renal failure onsets, while being very high for the 


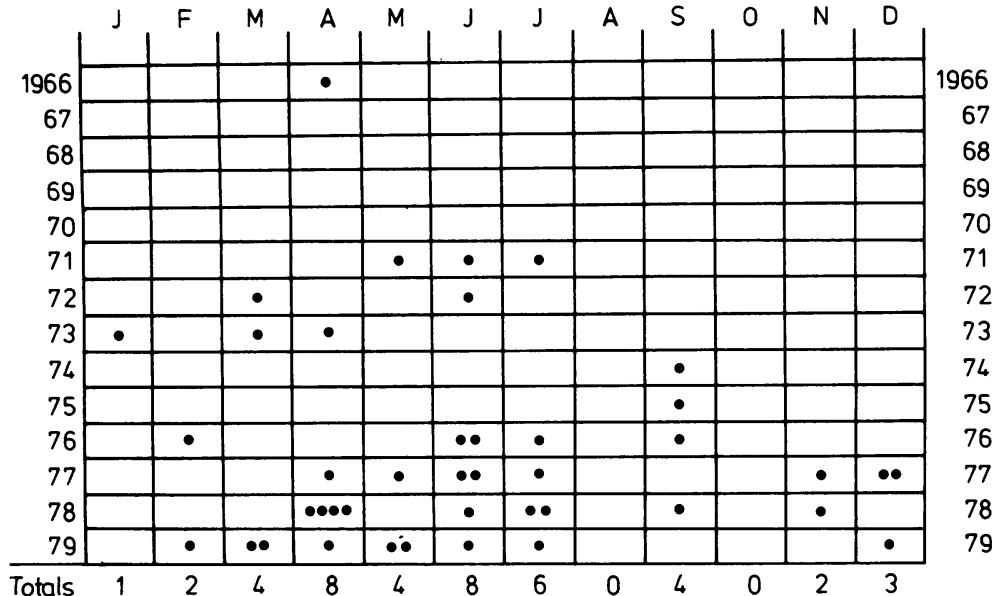

echovirus outbreak $(<0 \cdot 007)$. Similar failure to cluster in time was seen for primary biliary cirrhosis patients able to date onset to within a year only, and for asymptomatic patients by time of first diagnosis. Thus, on this analysis, primary biliary cirrhosis symptomatic onset was seasonal in a proportion of patients, but of low or absent epidemicity.

\section{PROXIMITY OF AFFECTED CASES}

Case clustering was determined in conurbation postal sectors. Analysis of pairs of patients appearing in the same sector shows the highest proportion occurring in echovirus notifications. The lowest proportion appears in renal failure and the proportion of primary biliary cirrhosis pairs does not differ significantly from it (Table). Thus clustering behaviour of primary biliary cirrhosis is closest to that of a non-infective disease.

\section{ANNUAL VARIATION IN FREQUENCY: RELATION TO} AGE AND PRESENTATION

Four main modes of presentation were identified: (i) symptomatic; with pruritis, jaundice, abdominal pain, or malaise; (ii) asymptomatic; as the result of incidental detection of hepatomegaly, positive mitochondrial antibody test or abnormal liver function tests; (iii) late or complicated; with portal hypertension or liver failure; and (iv) provoked. The last category of presentation was defined as clinical appearance of disease immediately in relation to one of the following factors: pregnancy, oestrogenic oral contraceptives, other drugs or major surgery other than for investigations of symptoms. Eleven patients $(9.4 \%)$ fell into this category: pregnancy (one patient), oestrogenic oral contraceptives (2), practolol (1), ${ }^{14}$ and non-investigative surgery with general anaesthesia (7). The numbers respectively of patients in these four main presentations are illustrated in Fig. 2, which shows that recruitment in all modes has tended to level off in recent years.

This suggests that maximal detection rate is being

Table (A) Proximity analysis: highest percentage of casepairs appearing within same postal sector belongs to high epidemicity (echovirus 19) patients, lowest to low epidemicity (renal failure). (B) Corresponding $\chi^{2}$ values: primary biliary cirrhosis expectation calculated from high and low epidemicity controls. Primary biliary cirrhosis is significantly closer in its geographical clustering behaviour to renal failure

(A) Proximity analysis: nos

\begin{tabular}{lllll} 
& $\begin{array}{l}\text { Conur- } \\
\text { bation } \\
\text { cases }\end{array}$ & $\begin{array}{l}\text { Total } \\
\text { case } \\
\text { pairs }\end{array}$ & $\begin{array}{l}\text { Same } \\
\text { postal } \\
\text { sector } \\
\text { pairs }\end{array}$ & $\%$ \\
\hline & $\mathrm{n}$ & $\mathrm{n}(\mathrm{n}-1) / 2$ & $\mathrm{n}^{\prime}$ & $\begin{array}{l}100 \times 2 \mathrm{n}^{\prime} / \\
\mathrm{n}(\mathrm{n}-1)\end{array}$ \\
$\begin{array}{c}\text { Controls } \\
\text { Low epidemicity } \\
\text { (renal) }\end{array}$ & 106 & 5565 & 31 & 0.56 \\
$\begin{array}{c}\text { High epidemicity } \\
\text { (echo) }\end{array}$ & 201 & 20100 & 213 & 1.06 \\
$\begin{array}{c}\text { Primary biliary } \\
\text { cirrhosis }\end{array}$ & 112 & 6216 & 41 & 0.66 \\
\hline
\end{tabular}

(B) Proximity analysis: $\chi^{2}$ values (dof $=1$ )

$$
\text { Controls }
$$

\begin{tabular}{lll}
\hline & Low & $\begin{array}{l}\text { High } \\
\text { epidemicity }\end{array}$
\end{tabular}

\begin{tabular}{llcc}
\hline $\begin{array}{l}\text { Primary biliary } \\
\text { cirrhosis }\end{array}$ & - & 0.92 & $9.39^{*}$ \\
$\begin{array}{l}\text { Controls } \\
\text { Low epidemicity }\end{array}$ & - & $12.46+$ \\
\hline
\end{tabular}

Inter-group differences significant at ${ }^{*} p<0.01,+p<0.0001$. 


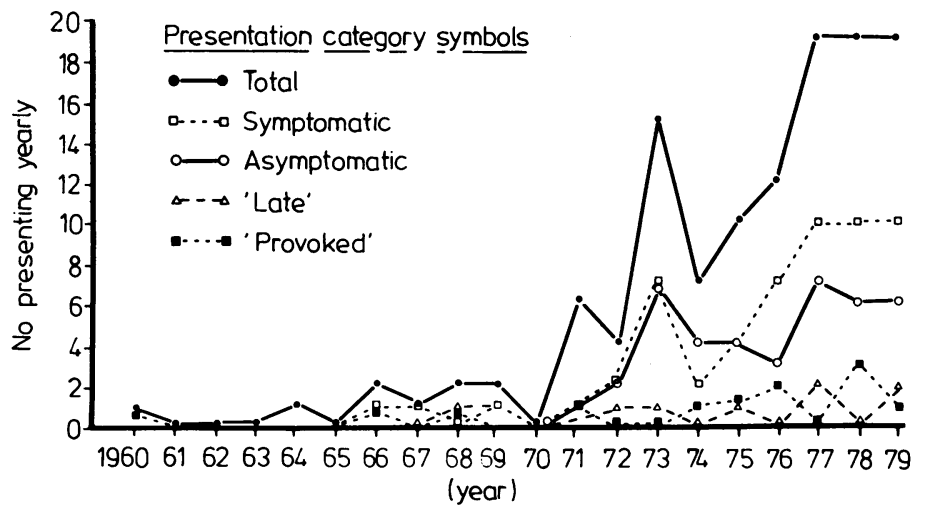

Fig. 2 Yearly occurrence of primary biliary cirrhosis. Over 1977-79 absolute numbers of various modes of onset have remained constant. achieved with the present methods and that asymptomatic individuals will eventually comprise between one third and one half of all primary biliary cirrhosis patients. It also shows that increased diagnosis of symptomatic disease is occurring, reflecting greater awareness of the condition. Age specific diagnosis rates rise significantly between ages 35 and 65 in all categories. Above age 65 prevalence exceeds $20 / 100000$ of women in this age group (Fig. 3).

\section{Discussion}

The Office of Population Censuses and Survey data show that over the last decade certified mortality from primary biliary cirrhosis in England and Wales has trebled. Even so, we were surprised to find our survey finding primary biliary cirrhosis, with a current annual diagnosis rate of $1 / 100000$, almost as common as Crohn's disease (1-3/100 000) and hepatobiliary carcinoma $(2-5 / 100000)$. Thus primary biliary cirrhosis, at least to gastroenterologists, is now a fairly frequent disease. Similar rates have been reported in other parts of Great Britain ${ }^{15}$ and the characteristics of our study area, with this geographical definition, low immigration rates and absence of other referral centres nearby support the reliability of these figures.

A previous mortality survey ${ }^{16}$ was unable to define local differences in primary biliary cirrhosis occurrence and we could not confirm recent reports of case clustering or a relationship to water supply. ${ }^{15}$ The River Tyne divides the main conurbation into two principal reservoir areas, but districts of high and low prevalence were found on either bank. These exhibited the varying ratios of asymptomatic to total cases which we have ascribed to differing diagnostic habits. Standards of medical care and practice are, therefore, the dominant factors in determining the subregional distribution of primary biliary cirrhosis.

This study does not, of course, exclude important

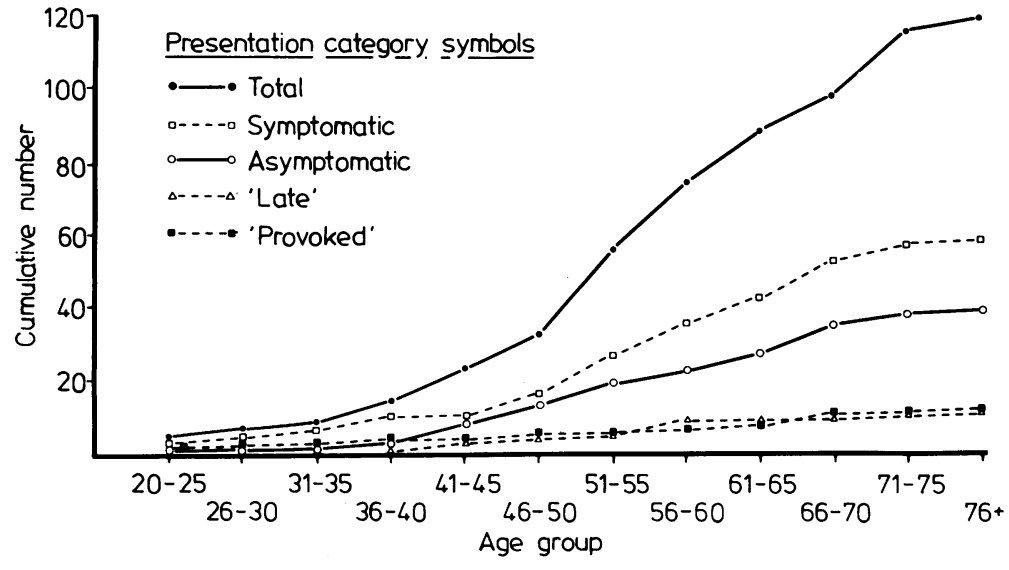

Fig. 3 Cumulative primary biliary cirrhosis presentation by age. Age-specific rates rise increasingly in middle life for all categories. 
supra-regional differences in the incidence of primary biliary cirrhosis. There is an impression (which remains to be confirmed by systematic survey) that real differences exist within Europe, with low rates in the Mediterranean countries and high rates in Great Britain and Scandinavia.

It is difficult to invoke racial or genetic factors as an explanation for the differences. In our series the relative incidence of affected kin was less than $1 \%$ and surveys of blood groups ${ }^{17}$ and HLA antigens ${ }^{18} 19$ have yielded negative results.

The validity of analysing symptomatic onset may be questioned. Only a minority of patients were able to define the date of appearance of first symptoms accurately, and the latent period of presymptomatic primary biliary cirrhosis is unknown. Short of longitudinal population studies, however, no other practical approach exists. Amelioration of the pruritus of primary biliary cirrhosis by ultraviolet light has been described ${ }^{20}$ and our findings of a deficit of symptomatic cases in the late summer months suggest that a sunny climate may affect the mode of presentation.

A striking observation in this series is the existence of high age-specific prevalence rates in women over 65 years. In fact, although median age at first presentation was 56 years, $27 \%$ of all patients were over this age at diagnosis. Twelve deaths $(67 \%$ of total mortality) were in this age group. Thus primary biliary cirrhosis now affects an older group than previously thought. ${ }^{2}$

Because of the female predominance of our series, occupational, and socio-economic factors were not analysed in great detail. Mortality data ${ }^{16}$ originally suggested that primary biliary cirrhosis was more frequent in higher socio-economic groups. This is probably not the case with our data, as some of the highest prevalence rates were found in industrial areas where manual and allied occupations prevailed. More detailed studies of the effect of occupation, housing, and other social conditions upon the occurrence of primary biliary cirrhosis are needed to decide the aetiology of this puzzling disease.

We acknowledge the cooperation of Dr T Bird, of the Newcastle General Hospital Immunology Laboratory, of $\operatorname{Dr} A$ A Codd of the Newcastle Public Health Laboratory, and Dr M Ward of the Department of Renal Medicine. The Office of Population Censuses and Surveys and the PO Postcode Section were especially helpful and Dr J C Dewdney of Durham University gave valuable cartographic advice. The cooperation of regional physicians, including Drs E A Cameron, S Roberts, and D Gooptu was particularly appreciated. Mrs S J
Hubble typed the manuscript and Miss $\mathrm{J}$ Taylor drew the figures. ANH was supported for part of the study by Newcastle AHA(T) Research Committee and Wellcome Trust grants.

\section{References}

1 Ahrens EH, Payne MA, Kunkel HG et al. Primary biliary cirrhosis. Medicine (Balt) 1950; 29: 299-364.

2 Sherlock S, Scheuer PJ. The presentation and diagnosis of 100 patients with primary biliary cirrhosis. $N$ Engl $J$ Med 1973; 289: 674-8.

3 Leevy CM, Tygstrup N. Standardisation of nomenclature, diagnostic criteria and diagnostic methodology for disease of the liver and biliary tract. In: Leevy CM, ed. Proc VI Quadr Meeting Internat Assoc Study of the Liver, Acapulco 1974. Basle: Karger, 1976.

4 Doniach D, Walker JG. Progress report: mitochondrial antibodies (AMA). Gut 1974; 15: 664-8.

5 Codd AA, Hale JH, Bell TM et al. Epidemic of echovirus 19 in the North-East of England. J Hyg (Camb) 1976; 76: 307-17.

6 The Post Office. Using postcodes in business systems. 2nd ed. London: Postcode Marking, 1979.

7 Central Statistical Office: Regional statistics No. 13. London: HMSO, 1977.

8 Office of Population Censuses and Surveys. Census 1971, England and Wales. County Report Series. London: HMSO, 1976.

9 Gilliam AG, McMahon B. Geographic distribution and trends of leukaemia in the United States. Acta Unio Internat Contr Cancr 1960; 16: 1623-8.

10 Knox G. Detection of low intensity epidemicity. Application to cleft lip and palate. Br J Prev Soc Med 1963; 17: 121-7.

11 Knox G. Epidemiology of childhood leukaemia in Northumberland and Durham. Br J Soc Prev Med 1964; 18: 17-25.

12 Wallenstein S. A test for detection of clustering over time. Am J Epidemiol 1980; 111: 367-72.

13 Freedman LS. The use of a Kolmogorov-Smirnov type statistics in testing hypotheses about seasonal variation. J Epidemiol Commun Hlth 1979; 33: 223-8.

14 Brown PJE, Lesna M, Hamlyn AN et al. Primary biliary cirrhosis after long-term practolol administration. Br Med J 1978; 1: 1591.

15 Triger DR. Primary biliary cirrhosis: an epidemiological study. Br Med J 1980; 281: 772-5.

16 Hamlyn AN, Sherlock S. The epidemiology of primary biliary cirrhosis: a survey of mortality in England and Wales. Gut 1974; 15: 473-9.

17 Hamlyn AN, Morris JS, Sherlock S. ABO blood groups, Rhesus negativity and primary biliary cirrhosis. Gut 1974; 15: 480-1.

18 Galbraith EM, Eddleston ALWF, Smith MGM et al. 
Histocompatibility antigens in active chronic hepatitis and primary biliary cirrhosis. $\mathrm{Br}$ Med J 1974; 3: 604-5.

19 Hamlyn AN, Adams D, Sherlock S. Primary or secondary sicca complex? Investigation in primary biliary cirrhosis by histocompatibility testing. $\mathrm{Br} \mathrm{Med} \mathrm{J}$ 1980; 281: 425-6.

20 Hanid MA, Levi AJ. Phototherapy for pruritis in primary biliary cirrhosis. (Abstract.) Gut 1980; 21: 447. 